\title{
Sevoflurane preconditioning prevents acute renal injury caused by ischemia-reperfusion in mice via activation of the Nrf 2 signaling pathway
}

\author{
WEN-XI WANG $^{1 *}$, ZHEN-RU ZHAO $^{1 *}$, YING BAI $^{1}$, YA-XING LI $^{1}$, \\ XIAO-NING GAO ${ }^{1}$, SEN ZHANG ${ }^{2}$ and YAN-BIN SUN ${ }^{1}$ \\ ${ }^{1}$ Department of Anesthesiology, Chengde Central Hospital, Chengde, Hebei 067000; \\ ${ }^{2}$ State Key Laboratory of Bioactive Substances and Functions of Natural Medicines, Institute of Materia Medica, \\ Chinese Academy of Medical Sciences and Peking Union Medical College, Beijing 100050, P.R. China
}

Received October 11, 2021; Accepted January 12, 2022

DOI: $10.3892 /$ etm.2022.11232

\begin{abstract}
Oxidative stress, caused by renal ischemia reperfusion (IR)/hypoperfusion, is one of the main causes of acute kidney injury (AKI). Previous studies have demonstrated that sevoflurane (SEV) protects organs from the damage caused by oxidative stress. In the present study, mice were randomly assigned to a sham operation group (Sham), IR-vehicle group (IR+ vehicle), IR + SEV low-dose preconditioning group and an IR + SEV high-dose preconditioning group. The effect of SEV on nuclear factor E2-related factor 2 (Nrf2), a key regulatory protein of the endogenous antioxidant defense system and, consequently oxidative stress, inflammation and apoptosis-related factors, were all quantified using commercial kits or by western blotting. SEV preconditioning was demonstrated to ameliorate kidney injury as a result of decreased blood urine nitrogen and serum creatinine levels, activated Nrf2 expression in the kidney and decreased oxidative stress and inflammatory index levels an AKI mouse model. SEV preconditioning also protected injured kidney via the downregulation of caspase-3 protein expression levels. In addition, using the Nrf2 inhibitor, Brusatol, significantly abolished the SEV preconditioning renal protective effect. Using
\end{abstract}

Correspondence to: Dr Yan-Bin Sun, Department of Anesthesiology, Chengde Central Hospital, 11 Guangren Street, Shuangqiao, Chengde, Hebei 067000, P.R. China

E-mail: sun0403@163.com

Professor Sen Zhang, State Key Laboratory of Bioactive Substances and Functions of Natural Medicines, Institute of Materia Medica, Chinese Academy of Medical Sciences and Peking Union Medical College, 2 Nanwei Road, Xicheng, Beijing 100050, P.R. China

E-mail: zhangs@imm.ac.cn

*Contributed equally

Key words: sevoflurane, acute kidney injury, ischemia reperfusion, nuclear factor E2 related factor 2, heme oxygenase-1 an in vitro HK-2 cell model of hypoxia/reoxygenation, it was also demonstrated that $\mathrm{Nrf} 2$ pathway activation was necessary for SEV to exert its beneficial effect for tubular cell injury caused by hypoxia/reoxygenation. These results indicated that SEV may protect against renal injury caused by IR via Nrf2 upregulation.

\section{Introduction}

Acute kidney injury (AKI) is a clinical syndrome, which presents with a rapid reduction in renal function over a short time period and is caused by numerous factors, including toxic agents, trauma or surgery $(1,2)$. It is characterized by a decreased glomerular filtration rate, retention of creatinine and urea nitrogen, a decrease in water and electrolytes, and impairment of acid-base balance. Severe AKI can also lead to multiple organ dysfunction syndromes $(1,3)$. Moreover, AKI causes huge social and economic burdens, especially in China (4). Renal ischemia reperfusion (IR)/hypoperfusion leads to the occurrence of oxidative stress and cell apoptosis, which is a direct result of decreased renal parenchymal cells, the atrophy of renal tissue structure and a decrease in renal function (renal insufficiency) (5). Surgical or pathological conditions, such as major vascular surgery, cardiac and hepatic surgeries, shock, sepsis, trauma, and kidney transplantation, can lead to IR-AKI (6). Currently, there is no effective method to reduce renal tissue injury and promote renal tissue repair. Renal tubular epithelial cell necrosis caused by renal hemodynamic changes is one of the pathophysiological mechanisms of AKI (2).

During apoptosis, several proteolytic enzyme families are activated, including caspases. Caspases are mainly activated via two signaling pathways: i) The exogenous death receptor signaling pathway and ii) the endogenous mitochondrial signaling pathway, which activate caspase- 8 zymogen and caspase- 9 zymogen, respectively. Both signaling pathways activate downstream effector caspase-3 leading to apoptosis (7). Therefore, caspase-3 is a terminal shear enzyme in the process of apoptosis. Chatterjee et al (8) reported that caspase inhibitors reduce the renal AKI response. Excessive 
levels of reactive oxygen species (ROS) induce apoptosis of functional cells. ROS cause tissue damage by triggering the caspase cascade, which includes activating caspase-3 and inducing cell apoptosis (9). Furthermore, renal tubular epithelial cells have an antioxidative defense system, which includes the nuclear factor-E 2-related factor 2 (Nrf2)/heme oxygenase-1 (HO-1)-mediated antioxidative response (10).

$\mathrm{Nrf} 2$ is a key regulatory protein in the endogenous antioxidant defense system. In oxidative stress, Nrf2 performs nuclear translocation and binds with the antioxidant response element (ARE) to start the transcription of numerous downstream antioxidant genes and serve a protective role in antioxidation (11). The antioxidant enzymes regulated by Nrf2 include superoxide dismutase (SOD), catalase (CAT), thioredoxin, peroxiredoxin, glutathione $(\mathrm{GSH})$ peroxidase, GSH reductase, glutamine cysteine ligase, glutamine cysteine synthase, $\mathrm{NAD}(\mathrm{P}) \mathrm{H}$ quinone oxidoreductase (NQO1) and HO-1 $(12,13)$.

Neutrophil gelatinase-associated lipocalin (NGAL) is a lipid carrier protein. It was originally discovered as a small molecular weight secretory protein in activated neutrophils (14). Under normal physiological conditions, NGAL is rarely expressed in the kidneys. When the renal tubular epithelium is damaged, a large amount of NGAL is secreted into the blood and urine (15). Therefore, neutrophils in the infiltrating tubulointerstitium are induced to transition to the tubules and the protein levels of NGAL in the urine and serum is increased (16). Thus, NGAL can be used as an effective biomarker for diagnosing AKI and early diabetic nephropathy $(17,18)$. Sevoflurane (SEV) is an inhaled anesthetic widely used in surgery. Previous studies have demonstrated that SEV has antioxidative stress and anti-inflammatory activity, and can therefore protect organs from damage caused by oxidative stress. Research over the past few years has demonstrated that SEV has different degrees of protection against renal injury $(19,20)$.

The present study therefore focused on the Nrf2 signaling pathway with the aim of studying the possible mechanism of SEV preconditioning on renal injury induced by IR in mice. Oxidative stress, the inflammatory response and apoptosis were all investigated.

\section{Materials and methods}

Animals. In total, 40 healthy 5-week-old male C57BL/6J mice (weight, $20 \pm 2$ g; SPF Biotechnology Co., Ltd.), were fed in cages and kept at $45 \%$ humidity and $20^{\circ} \mathrm{C}$ under a $12-\mathrm{h}$ light/dark cycle. All mice had free access to water and food. The mice were randomly assigned to the following groups: i) Sham operation group (Sham); ii) IR group + vehicle (IR + vehicle); iii) IR + SEV low-dose preconditioning (SEV-L; inhalation of $2.2 \% \mathrm{SEV}$ ); and iv) IR + SEV high-dose preconditioning (SEV-H; inhalation of $3.3 \% \mathrm{SEV}$ ). Each group contained 10 mice.

The second animal experiment aimed to further investigate the renal protective effect of SEV via activation of Nrf2. Another 40 healthy 5-week-old male C57BL/6J mice

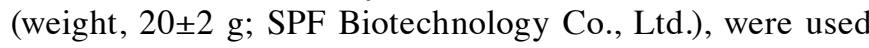
( $n=8$ mice/group). All mice were fed in cages and kept at $45 \%$ humidity and $20^{\circ} \mathrm{C}$ under a 12-h light/dark cycle, with free access to water and food. A further five groups of mice were involved: i) Sham group; ii) IR group; iii) IR + brusatol (Selleck Chemicals) group; iv) IR + SEV (high dose); and v) IR + SEV (high dose) + brusatol group. Brusatol (30 mg/kg), an Nrf2 inhibitor, was administered orally $2 \mathrm{~h}$ before preconditioning with SEV. Following IR, the levels of blood urea nitrogen (BUN) and serum creatinine (Scr) were detected.

IR surgery. The mice were anesthetized with an intraperitoneal injection of $1 \%$ sodium pentobarbital $(50 \mathrm{mg} / \mathrm{kg})$ and were fixed in the lateral position on the operating table. Longitudinal abdominal incisions from the left and right sides were cut until the kidneys were exposed. Both sides of the incision were covered with normal saline gauze. The renal pedicle capsule was peeled off and the separated renal artery on both sides was clamped with a non-invasive artery clamp. The kidney was returned in the abdominal cavity following successful clamping. The bilateral artery clamp was removed $45 \mathrm{~min}$ later. The kidneys were returned into their original place after confirming the recovery of blood perfusion. Subsequently, the muscle layer was sutured followed by the skin layer. All experimental animals maintained spontaneous breathing during the operation. The renal artery was not clamped in the Sham group, but all other experimental steps were the same as in the other groups. All animal experiments were conducted in strict accordance with the guidance provided on the treatment of experimental animals issued by the Ministry of Science and Technology of China (21). At the end of the experiments, all animals were euthanized by cervical dislocation.

Mice in the SEV group inhaled the mixture of low-dose or high-dose sevoflurane (Maruishi Pharmaceutical Co., Ltd.) and $\mathrm{O}_{2}(80 \%)$ for $60 \mathrm{~min}, 30 \mathrm{~min}$ prior to IR. The renal IR model was established following anesthesia. The mice were placed in a clean environment with a constant temperature of $22^{\circ} \mathrm{C}$ following surgery with free access to food and water. Urine was collected before mice were sacrificed by cervical dislocation.

Sample collection and serum marker detection. The mice were sacrificed $24 \mathrm{~h}$ following surgery. Blood samples were extracted from the heart, placed in a $1.5 \mathrm{ml}$ tube and centrifuged at $1,000 \times \mathrm{g}$ at $4^{\circ} \mathrm{C}$ for $15 \mathrm{~min}$ after standing at room temperature. The supernatant was taken for further analysis. BUN and Scr were quantified using an automatic biochemical instrument (TBA-120FR; Toshiba Corporation). NGAL levels were assessed using a commercially available ELISA kit (cat. no. ab119601; Abcam). Serum inflammatory cytokine levels, including TNF- $\alpha$ (cat. no. ab46105), IL-6 (cat. no. ab222503), and IL-1 $\beta$ (cat. no. ab197742) were also measured using ELISA kits (all purchased from Abcam). Intercellular adhesion molecule-1 (ICAM-1; cat. no. ab100688), monocyte chemoattractant protein-1 (MCP-1; cat. no. ab100721) and vascular cell adhesion protein-1 (VCAM-1; cat. no. ab201278) were measured using ELISA kits (all purchased from Abcam).

Renal histopathological evaluation. Renal tissues were fixed with $4 \%$ paraformaldehyde (PFA) at room temperature for $24 \mathrm{~h}$, embedded in paraffin, cut into $8-\mu \mathrm{m}$ thick sections, and stained with $\mathrm{H} \& \mathrm{E}$ at $35^{\circ} \mathrm{C}$ for $3 \mathrm{~min}$. The pathological changes in renal tissue were observed using a light microscope. A total of two different sections were taken from each 
sample to observe the histopathological changes of the kidney. According to the method introduced by Zhang et al $(22,23)$, for each animal, at least 10 high-power (magnification, x400) fields were examined. The percentage of tubules that displayed cellular necrosis, loss of brush border, cast formation, vacuolization and tubule dilation was scored as follows: 0 (none), $1(<10 \%), 2$ (11-25\%), 3 (26-45\%), 4 (46-75\%) and $5(>76 \%)$. Masson staining was performed at room temperature using a commercial staining kit (cat. no. G1340; Beijing Solarbio Science \& Technology Co., Ltd.) according to the manufacturer's protocol, to evaluate collagen fibrils in renal tissues. The staining was performed according to manufacturer's instruction. A total of 10 stained fields were randomly selected from each section and observed using a CX23 RFS2 LED light microscope (magnification, $\mathrm{x} 400$, Olympus Corporation). The images were analyzed by Image-Pro Plus 6.0 (Media Cybernetics, Inc.). For fibrotic area semi-quantification, a ratio of blue stained area to the area of the entire field (including glomeruli, tubule lumina and blood vessels) was assessed and expressed as a percentage of Masson staining.

Detection of oxidative stress. A total of $0.2 \mathrm{~g}$ kidney tissue was ground, centrifuged at $1,000 \times \mathrm{g}$ for $10 \mathrm{~min}$ at $4^{\circ} \mathrm{C}$. The supernatant was obtained to detect the concentrations of different proteins. The enzyme activity of SOD (cat. no. A001-3), myeloperoxidase (MPO; cat. no. A044) and CAT (cat. no. A007), as well as the concentration of malondialdehyde (MDA; cat. no. A003-1) were determined using commercial kits (all purchased from Nanjing Jiancheng Bioengineering Institute) according to the manufacturer's protocol.

Immunohistochemistry. Immunohistochemistry was performed using a two-step method. Briefly, 5- $\mu \mathrm{m}$ thick sections of mouse kidney were dewaxed and rehydrated using xylene and gradient alcohol, treated with $1 \%$ hydrogen peroxide solution at $37^{\circ} \mathrm{C}$ for $10 \mathrm{~min}$, heated and repaired using an antigen repair solution (1:10; cat. no. P0088; Beyotime Institute of Biotechnology), cooled to room temperature and incubated with primary and secondary antibodies. The slide was incubated with DAB solution (cat. no. P0203; Beyotime Institute of Biotechnology) for $8 \mathrm{~min}$ at room temperature and then sealed for observation. Positive staining was indicated by yellowish-brown tissues. The following primary antibodies were used: Caspase-3 (1:100; cat. no. 66470-2-Ig), Nrf2 (1:100; cat. no. 66504-1-Ig), HO-1 (1: 100; cat. no. 27282-1-AP) and NQO1 (1:100; cat. no. 67240-1-Ig) (all from ProteinTech Group, Inc.). The sections were examined using a light microscope (DMLS-1000; Leica Microsystems GmbH) with a high-power field of view (magnification, x400). A total of five visual fields were randomly selected and the images were analyzed using Biosens Digital Imaging System Software version 16 (Shanghai Bio-Tech Co., Ltd.). The color of the images was transformed to separate the positive areas from the background for automatic measurement. The following formula was used: Positive target expression index $(\%)=$ average optical density of positive target $\mathrm{x}$ positive area/(positive area + negative area).

TUNEL staining. The kidney sections from each group were dewaxed with xylene and washed with $100 \%$ ethanol, rehydrated and washed in decreasing concentrations of ethanol and were immersed in $0.85 \% \mathrm{NaCl}$, then washed twice with PBS. Apoptosis was detected in tissue sections using the DeadEnd ${ }^{\mathrm{TM}}$ Colorimetric Apoptosis Detection System (Promega Corporation). Samples were fixed with $4 \%$ PFA for $15 \mathrm{~min}$ at $20-25^{\circ} \mathrm{C}$ and immersed in PBS twice for $10 \mathrm{~min}$. Subsequently, samples were permeabilized with $20 \mu \mathrm{g} / \mathrm{ml}$ proteinase $\mathrm{K}$ solution and incubated for $20 \mathrm{~min}$ at room temperature. Slides were washed in PBS and fixed with 4\% PFA at room temperature for $5 \mathrm{~min}$, washed, and equilibrated with equilibration buffer for $10 \mathrm{~min}$. Then, $100 \mu \mathrm{l}$ terminal deoxynucleotidyl transferase mix was added to the tissue sections, which were incubated for $60 \mathrm{~min}$ at $37^{\circ} \mathrm{C}$ and then washed in PBS. The slides were immersed in $0.3 \%$ hydrogen peroxide for $3 \mathrm{~min}$ and washed in PBS. Subsequently, $100 \mu \mathrm{l}$ streptavidin HRP (cat. no. A0303; 1:500; in PBS; Beyotime Institute of Biotechnology), was added and the samples were incubated for $30 \mathrm{~min}$ at room temperature. After the slides were washed in PBS, $100 \mu \mathrm{l}$ DAB solution (Beyotime Institute of Biotechnology) was added to stain the slides for $10 \mathrm{~min}$ at $15-25^{\circ} \mathrm{C}$. The slides were washed in deionized water and were mounted using mounting medium. TUNEL-positive apoptotic cells were visualized using a light microscope (magnification, x200).

Western blotting. After homogenizing the preserved kidney tissues, total protein was extracted using a protein extraction kit (Beijing Solarbio Science \& Technology Co., Ltd.) and the protein concentration was quantified using the BCA method. Total protein, using $30 \mu \mathrm{g}$ from each sample, was separated using $10 \%$ SDS-PAGE and transferred onto a PVDF membrane. The membranes were blocked with $5 \%$ skimmed milk for $1.5 \mathrm{~h}$ at $20-25^{\circ} \mathrm{C}$, incubated overnight at $4^{\circ} \mathrm{C}$ with primary antibodies and were subsequently incubated with a suitable secondary antibody at room temperature for $1 \mathrm{~h}$. An Enhanced Chemiluminescence Kit (Amersham; Cytiva) was used to detect protein bands. $\beta$-actin served as a loading control. Bands were semi-quantified via scanning densitometry using the Odyssey ${ }^{\circledR}$ CLx Imaging System and Image Studio Version 3.1 software provided by the manufacturer (LI-COR Biosciences). The primary antibodies included caspase-3 (cat. no. 66470-2-Ig; 1:1,000), Nrf2 (cat. no. 66504-1-Ig; 1: 1,000), HO-1 (cat. no. 27282-1-AP; 1:1,000) and NQO1 (cat. no. 67240-1-Ig; 1:1,000) (all from ProteinTech Group, Inc.). HRP goat anti-mouse IgG (1:5,000; cat. no. sc-2005) or HRP goat anti-rabbit IgG (1:4,000; cat. no. sc-2054) (both from Santa Cruz Biotechnology, Inc.) was used.

Cell culture and in vitro HK-2 cell model of hypoxia/reoxygenation $(H / R)$. The human renal proximal tubular HK-2 cell line was purchased from American Type Culture Collection. Cells were plated in 100-mm culture dishes and cultured in DMEM supplemented with 10\% FBS (both from Thermo Fisher Scientific, Inc.), $100 \mathrm{U} / \mathrm{ml}$ penicillin, $2 \mathrm{mM}$ glutamine, $100 \mu \mathrm{g} / \mathrm{ml}$ streptomycin and $1 \mathrm{mM}$ HEPES buffer. Cells were cultured at $37^{\circ} \mathrm{C}$ in humidified air containing $5 \% \mathrm{CO}_{2}$. The medium was replaced every other day. For the H/R group, cells were exposed to hypoxic conditions $\left(5 \% \mathrm{CO}_{2}, 1 \% \mathrm{O}_{2}\right.$ and $\left.94 \% \mathrm{~N}_{2}\right)$ for $24 \mathrm{~h}$ followed by $12 \mathrm{~h}$ of reoxygenation $\left(5 \% \mathrm{CO}_{2}, 21 \% \mathrm{O}_{2}\right.$ and $\left.74 \% \mathrm{~N}_{2}\right)$. To determine the effect of SEV, cells were pretreated with either vehicle buffer (0.5\% DMSO solution) or SEV 


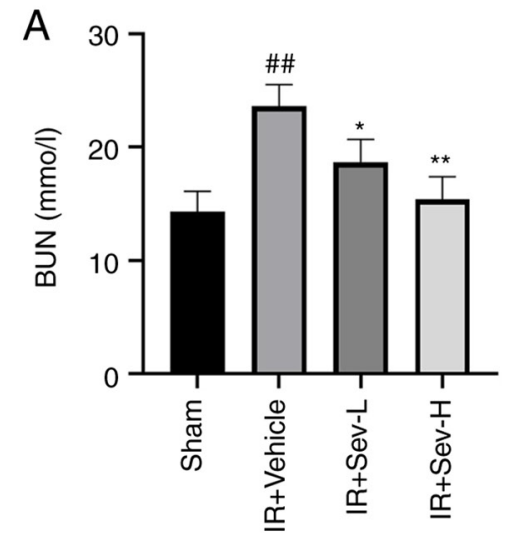

B

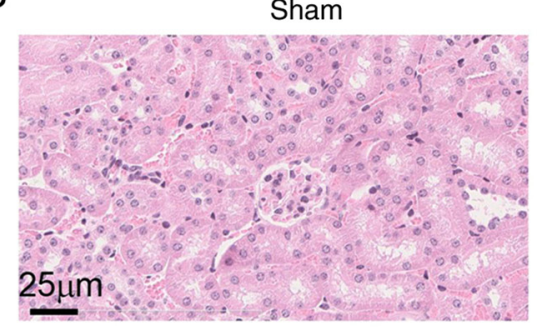

IR+Sev-L

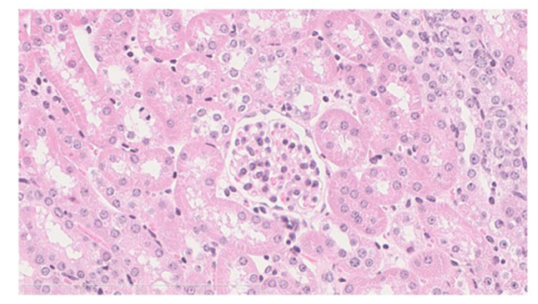

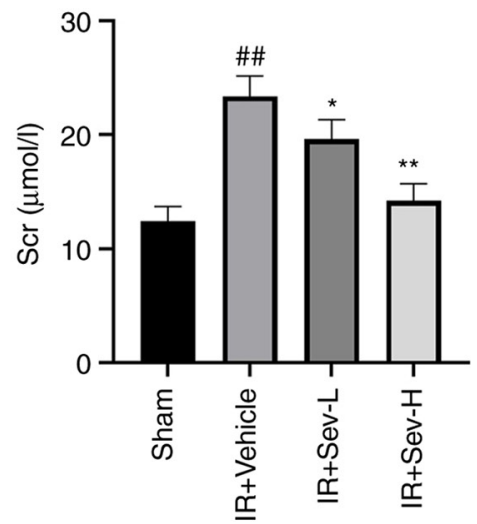

IR+Vehicle

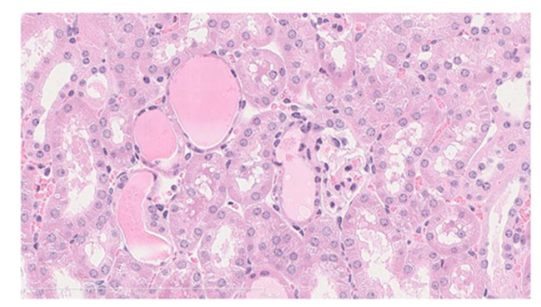

$\mathrm{IR}+\mathrm{Sev}-\mathrm{H}$

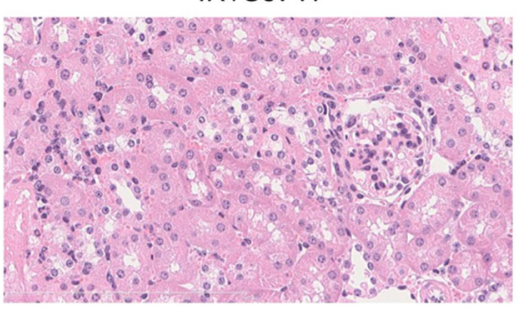

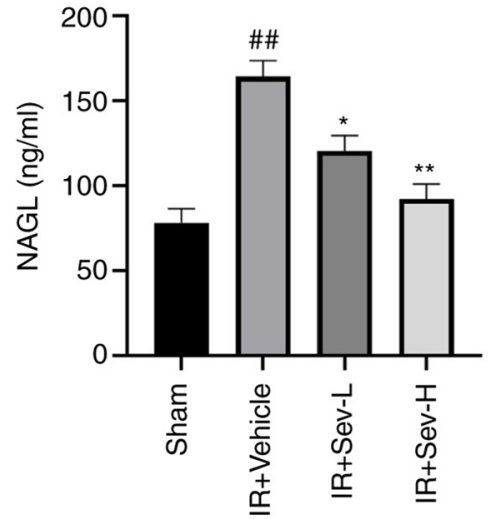

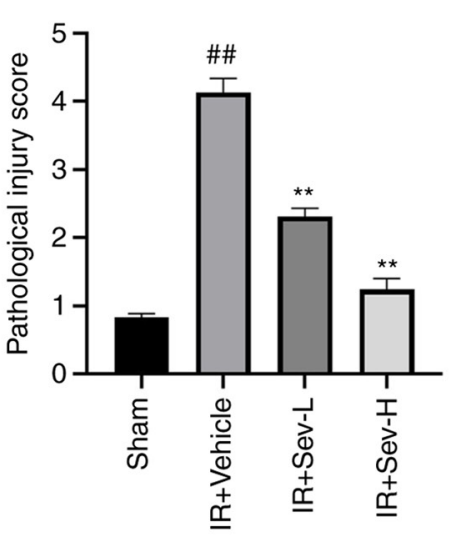

Figure 1. SEV decreases BUN, Scr and NGAL levels and improves the pathological damage caused by IR. (A) SEV preconditioning decreased the BUN, Scr and serum NGAL levels. (B) High-dose SEV preconditioning improved acute tubular injury induced by IR. ${ }^{\# \#} \mathrm{P}<0.05$ vs. sham; ${ }^{* *} \mathrm{P}<0.01,{ }^{*} \mathrm{P}<0.05$ vs. IR vehicle control. SEV, sevoflurane; BUN, blood urine nitrogen; Scr, serum creatinine; NGAL, neutrophil gelatinase-associated lipocalin; IR, ischemia reperfusion.

( 1 and 2 concentration) for $12 \mathrm{~h}$ prior to $\mathrm{H} / \mathrm{R}$ treatment. A Nrf2 inhibitor, brusatol (10 $\mathrm{nM})$ was also added to evaluate whether SEV, serving its protective role, was dependent on the Nrf2 signaling pathway. The in vitro study contained the following six groups; i) Control group without H/R; ii) $H / R$ group treated with vehicle; iii) $H / R$ group treated with $1 \% \mathrm{SEV}$; iv) $\mathrm{H} / \mathrm{R}$ group treated with $2 \% \mathrm{SEV}$; v) H/R group treated with brusatol; and vi) H/R group treated with brusatol and 2\% SEV. An in-line anesthetic vaporizer (Drägerwerk AG \& Co., KGaA) fed by a supply of a gas containing $21 \% \quad \mathrm{O}_{2}$ and $5 \% \quad \mathrm{CO}_{2}$, balanced with $\mathrm{N}_{2}$, was used to deliver SEV at a rate of $21 / \mathrm{min}$ for at least $5 \mathrm{~min}$ until the desired SEV concentration (1-2\%) was achieved. Concentrations of SEV and $\mathrm{O}_{2}$ were monitored every $\mathrm{h}$ using an anesthetic analyzer (Drägerwerk AG \& Co. $\mathrm{KGaA}$ ). Cells in the vehicle control conditions were placed in an identical gas chamber under $21 \% \mathrm{O}_{2}$ and $5 \% \mathrm{CO}_{2}$, balanced with $\mathrm{N}_{2}$. Once sealed, the chambers were placed in a $37^{\circ} \mathrm{C}$ incubator.

Cell viability assay. HK-2 cells were treated with Cell Counting Kit-8 reagent (10 $\mu \mathrm{l} /$ well; Applygen Technologies, Inc.) for $2 \mathrm{~h}$. The optical density was recorded at $450 \mathrm{~nm}$ using a microplate absorbance reader (Tecan Group, Ltd.).
Statistical analysis. The data were analyzed using GraphPad Prism 8.0 software (GraphPad Software, Inc.). Data are presented as the mean \pm SD. Statistical comparisons among the groups were analyzed using one-way ANOVA followed by Bonferroni's post hoc test. $\mathrm{P}<0.05$ was considered to indicate a statistically significant difference.

\section{Results}

Renalfunction. Compared with that in sham group, the concentration of BUN, Scr, and serum NGAL in the IR group were significantly increased (all $\mathrm{P}<0.01$ ) (Fig. 1A). Preconditioning with SEV decreased the BUN, Scr and serum NGAL concentrations in a dose-dependent manner (Fig. 1A). The H\&E staining (Fig. 1B) also demonstrated that preconditioning with SEV attenuated acute renal tubular injury induced by $I / R$ in a dose-dependent manner, consistent with the biochemical analysis.

SEV preconditioning decreases tubular cell apoptosis and cleaved caspase-3 protein expression levels. It was determined via TUNEL staining that preconditioning with SEV resulted in a significantly reduced quantity of apoptotic tubular cells in the renal interstitium compared with that in 
A
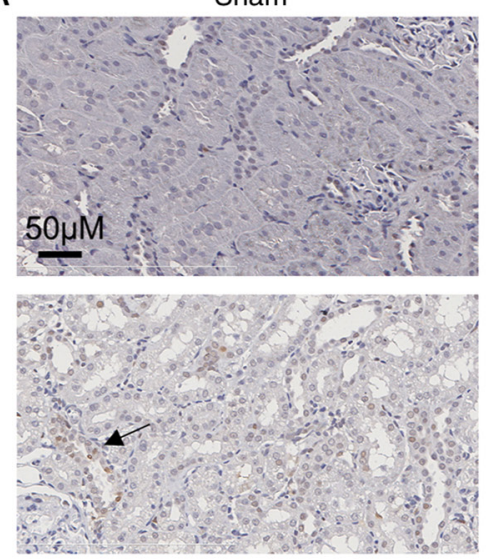

$\mathrm{IR}+\mathrm{Sev}-\mathrm{L}$

B
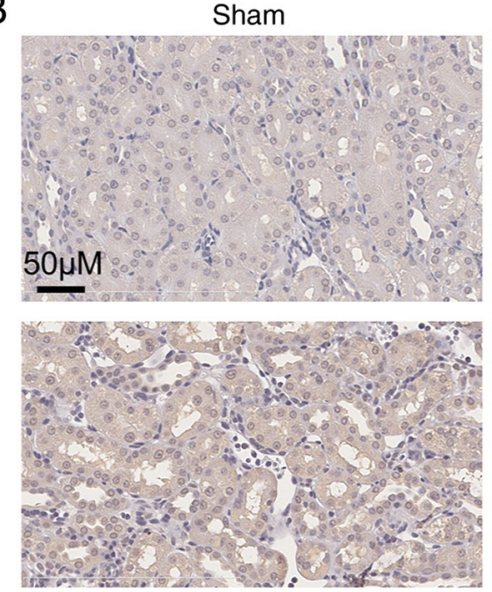

$\mathrm{IR}+\mathrm{Sev}-\mathrm{L}$

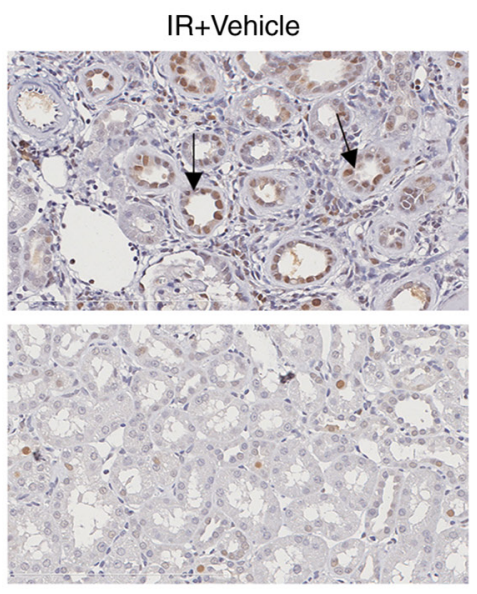

$\mathrm{IR}+\mathrm{Sev}-\mathrm{H}$
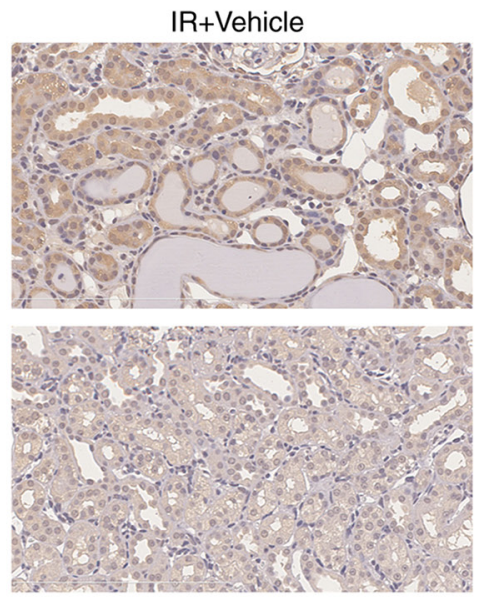

$\mathrm{IR}+\mathrm{Sev}-\mathrm{H}$
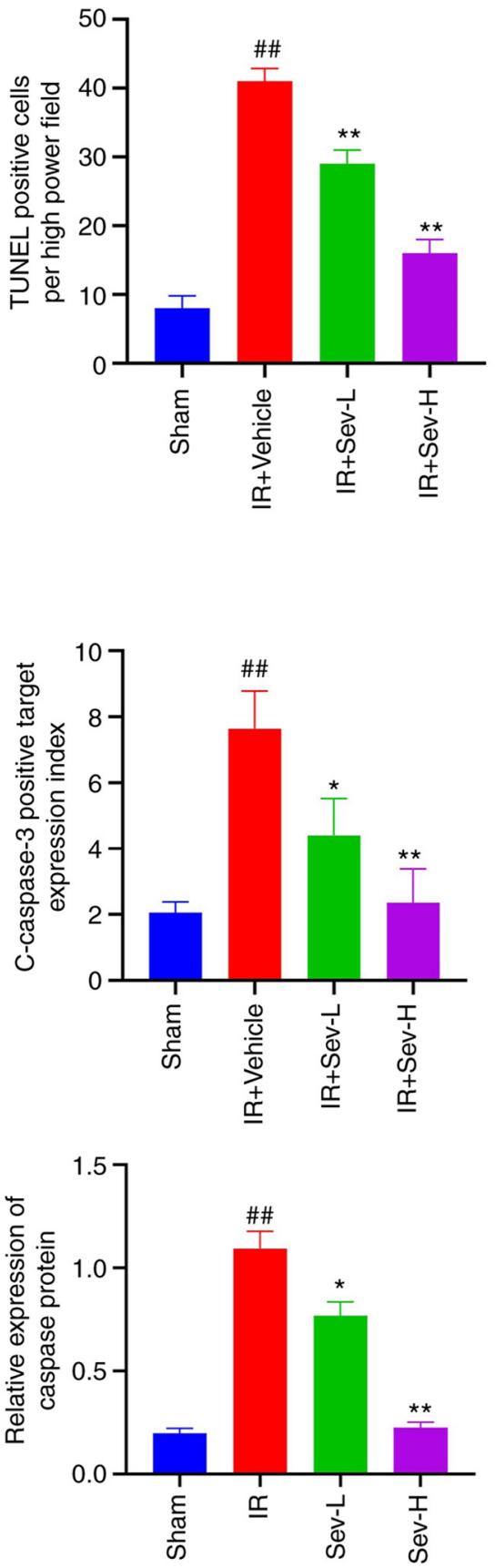

Figure 2. SEV preconditioning decreases tubular cell apoptosis and reduces cleaved caspase-3 expression in impaired tubular cells. (A) TUNEL staining was performed to determine apoptotic cells. The arrow refers to TUNEL positive cells (magnification, $\mathrm{x} 200$ ). (B) Immunohistochemical staining of cleaved caspase-3 in different groups left and quantification of the cleaved caspase-3 (right). (C) Western blot analysis of caspase-3 protein expression levels in the different IR groups were significantly higher than those in the sham group. ${ }^{\# \#} \mathrm{P}<0.05$ vs. sham; ${ }^{* *} \mathrm{P}<0.01$, ${ }^{*} \mathrm{P}<0.05$ vs. IR vehicle control. SEV, sevoflurane; IR, ischemia reperfusion; L, low; $\mathrm{H}$, high; $\mathrm{C}$, cleaved.

the vehicle control group $(\mathrm{P}<0.05$; Fig. $2 \mathrm{~A})$. Actively cleaved caspase-3 protein was mainly expressed in the cytoplasm and nucleus of impaired renal tubular epithelial cells. The results demonstrated that the protein expression level of cleaved caspase-3 in the sham group was low, whereas IR-induced AKI increased the caspase- 3 expression levels in the cytoplasm and nucleus of renal tubular epithelial cells. SEV preconditioning significantly decreased the caspase- 3 protein expression levels in a dose-dependent manner, especially in the SEV-H group (Fig. 2B). Western blotting (Fig. 2C) also revealed that SEV preconditioning reduced cleaved caspase-3 protein expression levels compared with that in the IR vehicle group $(\mathrm{P}<0.01)$.

SEV preconditioning alleviates inflammation and oxidative stress. The results demonstrated that IR led to the increased levels of cytokines and chemokines, including TNF- $\alpha$, IL- 6 , IL-1 $\beta$, ICAM-1, MCP-1 and VCAM- 1 compared with in the 

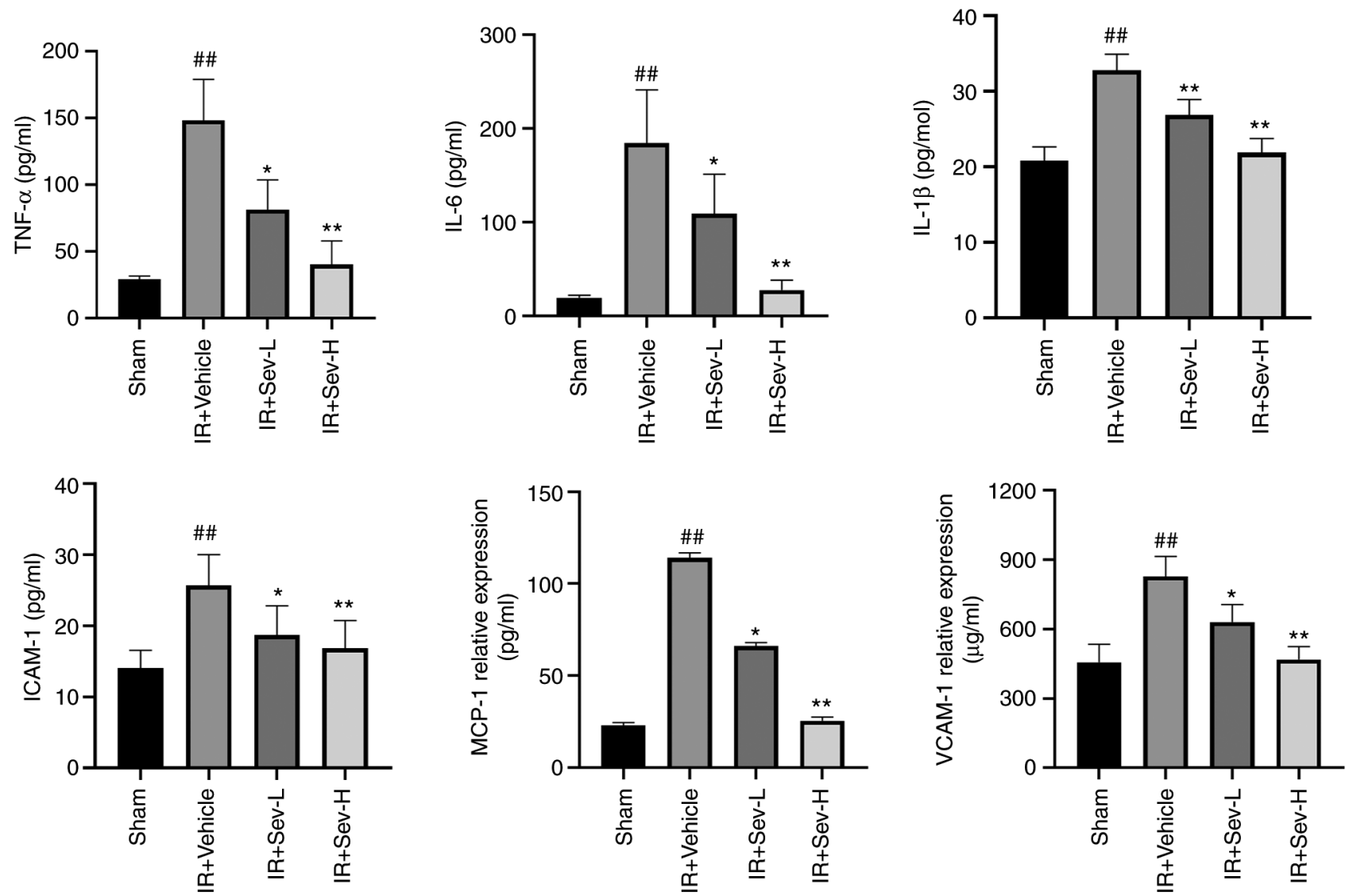

Figure 3. SEV preconditioning significantly reduces cytokine and chemokine levels. The concentration of inflammatory factors TNF- $\alpha$, IL-6, IL-1 13 ICAM-1, MCP-1 and VCAM-1 in the peripheral blood were determined by ELISA kits. ${ }^{\# \#} \mathrm{P}<0.05$ vs. sham; ${ }^{* *} \mathrm{P}<0.01,{ }^{*} \mathrm{P}<0.05$ vs. IR vehicle control. SEV, sevoflurane; MCP-1, monocyte chemoattractant protein-1; ICAM-1, intercellular adhesion molecule-1; VCAM-1, vascular cell adhesion protein-1; IR, ischemia reperfusion; L, low; H, high.
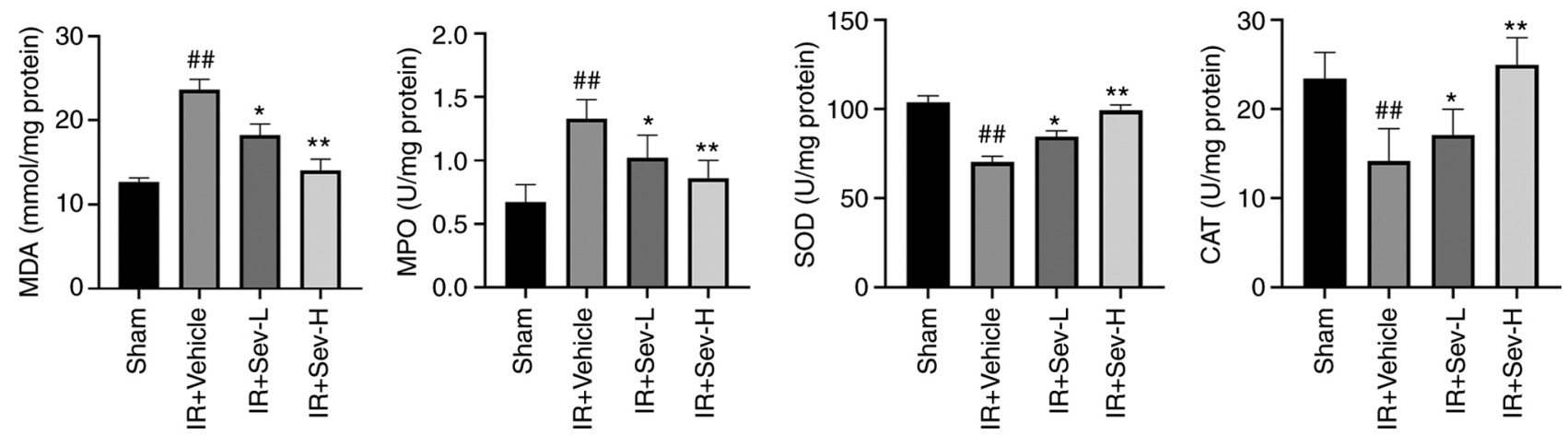

Figure 4. Effect of SEV preconditioning on oxidative stress. Quantitative analysis indicate the concentrations of MDA and MPO, as well as SOD and CAT in the kidney tissues from IR mouse model. ${ }^{\# \#} \mathrm{P}<0.05$ vs. sham; ${ }^{* *} \mathrm{P}<0.01,{ }^{*} \mathrm{P}<0.05$ vs. IR vehicle control. SEV, sevoflurane; MDA, malondialdehyde; MPO, myeloperoxidase; SOD, superoxide dismutase; CAT, catalase; IR, ischemia reperfusion; L, low; $\mathrm{H}$, high.

sham group (Fig. 3). SEV preconditioning significantly reduced cytokine and chemokine levels in the peripheral blood compared with in the IR + vehicle group, especially in the SEV-H group. Similarly, SEV preconditioning significantly decreased concentrations of MDA and MPO, whereas the activity of SOD and CAT was significantly increased as shown in Fig. 4.

SEV preconditioning activated the expression of $N r f 2$ in the kidney. Due to the antioxidant properties of SEV preconditioning as demonstrated in the aforementioned results, it was hypothesized that SEV preconditioning might activate the endogenous antioxidant defense system and that the Nrf2 signaling pathway was a possible target. By performing immunohistochemistry it was determined that the Nrf2 protein expression levels in kidney tissue in the preconditioning groups were notably increased compared with that in the IR group. Moreover, its downstream proteins HO-1 and NQO-1 were also notably upregulated by SEV preconditioning (Fig. 5A). Western blotting demonstrated a similar trend to the immunohistochemistry results (Fig. 5B), which further confirmed that 
A
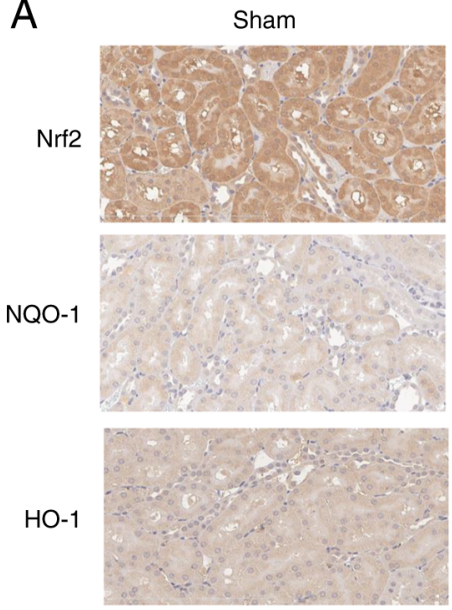

IR+Vehicle
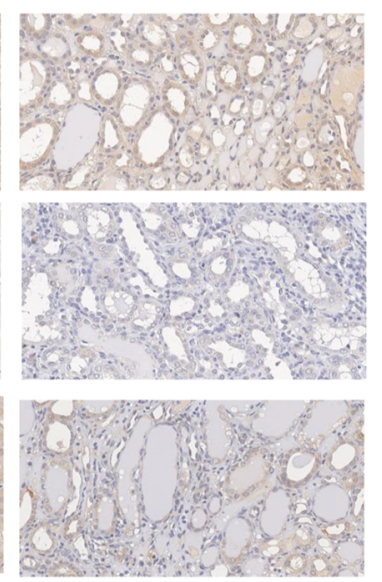

IR+Sev-L
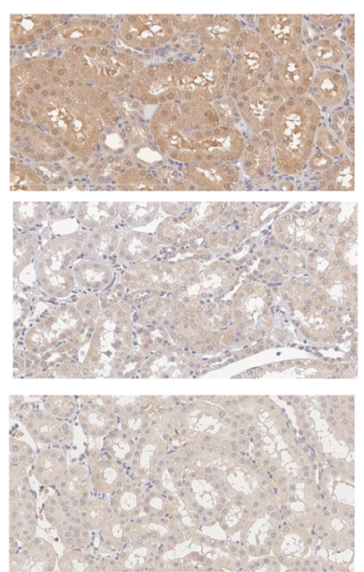

$\mathrm{IR}+\mathrm{Sev}-\mathrm{H}$
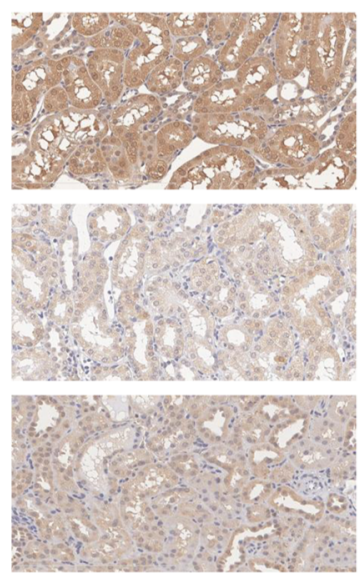

B
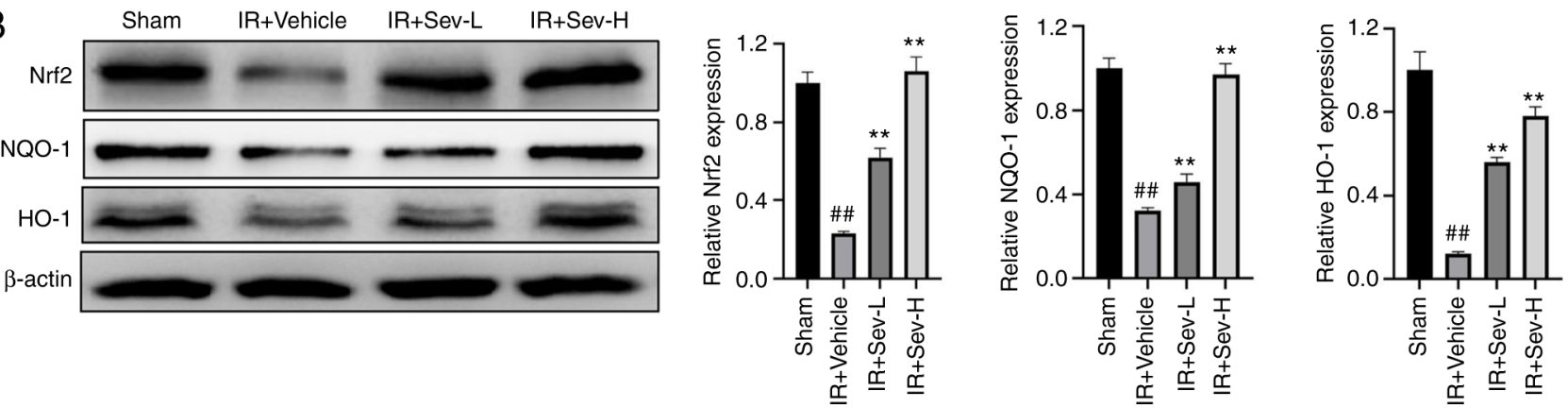

Figure 5. SEV preconditioning activates the Nrf2/HO-1 signaling pathway. (A) Immunohistochemistry was used to detect the protein expression levels of Nrf2 and its downstream proteins HO-1 and NQO-1 in different IR groups. (B) Protein expression levels of Nrf2, HO-1 and NQO-1 in kidney tissues were determined using western blot analysis. ${ }^{\# \#} \mathrm{P}<0.05$ vs. sham; ${ }^{* *} \mathrm{P}<0.01$ vs. IR vehicle control. SEV, sevoflurane; Nrf2, nuclear factor E2-related factor 2; HO-1, heme oxygenase-1; NQO-1, NAD(P)H quinone oxidoreductase; IR, ischemia reperfusion; L, low; H, high.

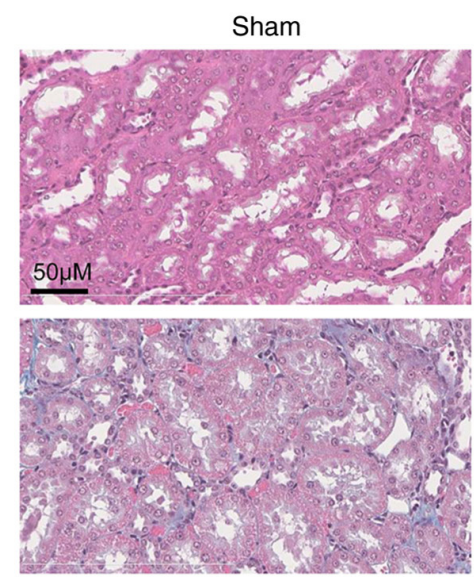

IR+Sev-L

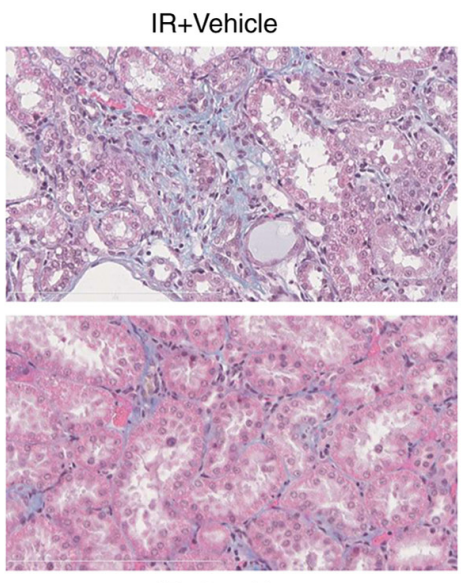

IR+Sev-H

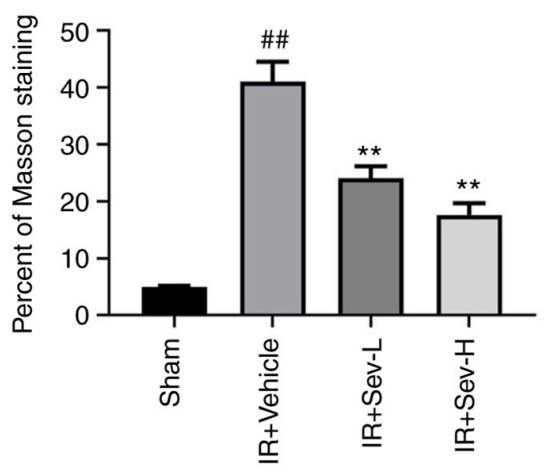

Figure 6. SEV preconditioning improves renal fibrosis caused by IR. Masson staining on mouse kidney sections in different SEV groups (left) and quantification bar graph (right). ${ }^{\# \#} \mathrm{P}<0.05$ vs. sham; ${ }^{* *} \mathrm{P}<0.01$ vs. IR vehicle control. SEV, sevoflurane; IR, ischemia reperfusion; $\mathrm{L}$, low; H, high.

SEV preconditioning may activate the Nrf2 signaling pathway in the kidneys from the mouse model of IR.

SEV preconditioning improves renal fibrosis caused by IR. In clinical practice, IR may cause renal interstitial fibrosis in the later stages and consequently, may affect the prognosis of AKI. A similar situation was discovered in animal models (24). In the present study, IR resulted in significant fibrosis of the renal interstitium in mice two weeks after IR, which was assessed using the ratio of Masson staining in high resolution fields (Fig. 6). As well as fibrosis, widening of the renal interstitium and atrophy of renal tubules were also evident in the microscopic sections. The degree of fibrosis was significantly reduced following SEV preconditioning, as well as amelioration of widening of the renal interstitium and atrophy of renal tubules. 


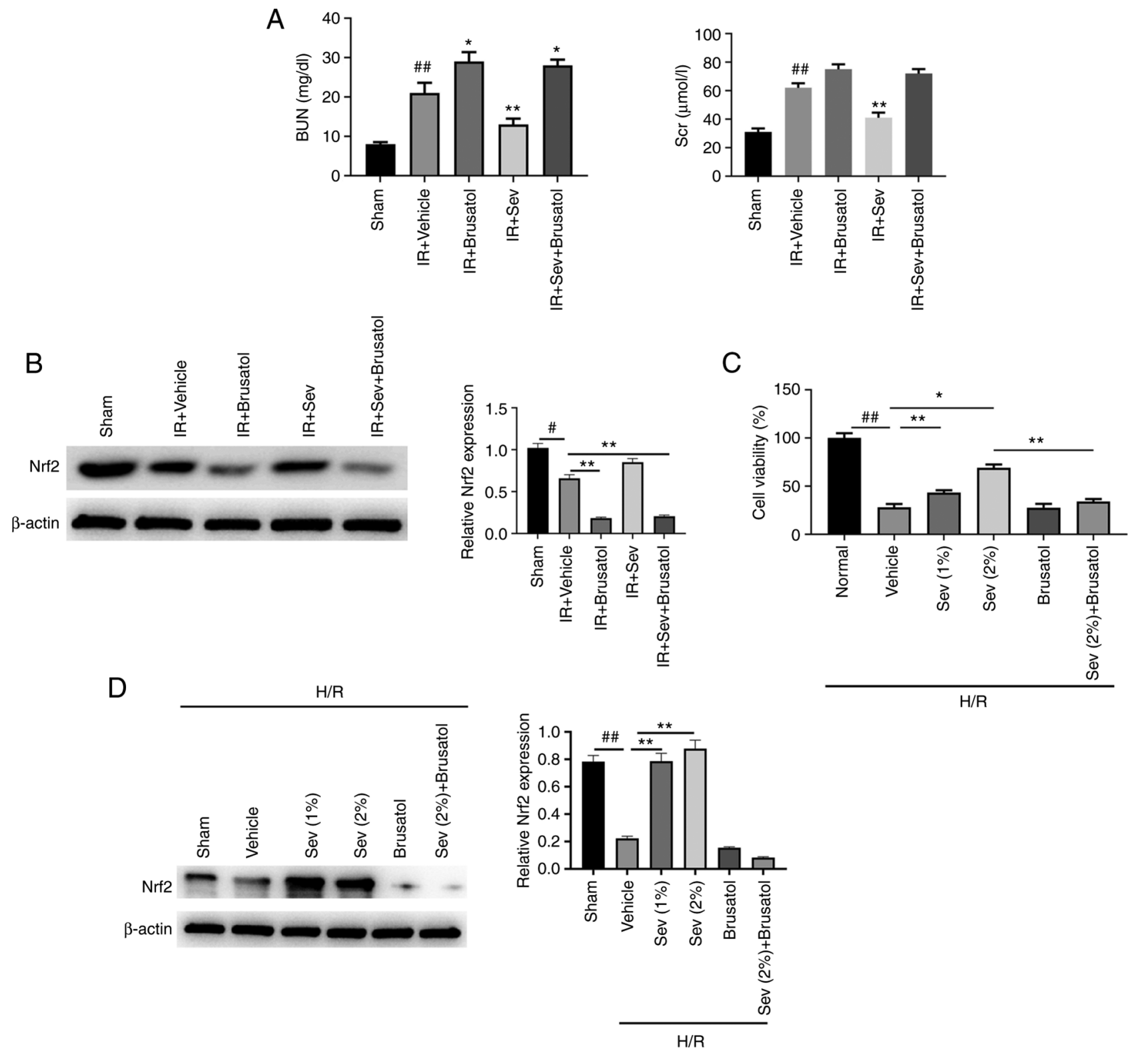

Figure 7. SEV does not exert renal protection in vivo and in vitro following Nrf2 signaling pathway inhibition by brusatol. (A) BUN and Scr levels following IR and co-treatment with brusatol. (B) Nrf2 protein expression levels in kidney tissues from each group were quantified using western blot analysis. (C) In vitro HK2 cell viability assay in the H/R model. (D) Nrf2 protein level in HK2 cells from each group were quantified using western blot analysis. ${ }^{* *} \mathrm{P}<0.01,{ }^{*} \mathrm{P}<0.05$ vs. IR vehicle control; ${ }^{\# \#} \mathrm{P}<0.01,{ }^{\#} \mathrm{P}<0.05$ vs. sham. SEV, sevoflurane; Nrf2, nuclear factor E2-related factor 2; BUN, blood urine nitrogen; Scr, serum creatinine; IR, ischemia reperfusion; $\mathrm{H} / \mathrm{R}$, hypoxia/reoxygenation.

Renal protective effect of SEV decreases following Nrf2 signaling pathway inhibition both in vivo and in vitro. To further confirm the renal protective effect of SEV via the activation of Nrf2, another five groups of animal experiments were conducted. Brusatol $(30 \mathrm{mg} / \mathrm{kg})$, an Nrf2 inhibitor, was orally administered $2 \mathrm{~h}$ prior to SEV preconditioning to the allocated groups. Following IR, the BUN and Scr levels were detected. The results demonstrated that brusatol administration slightly aggravated renal injury in IR mice (Fig. 7A). Moreover, the renal protective effect of SEV was almost abolished by pretreatment with brusatol. The Nrf2 protein expression levels in kidney tissues were significantly reduced by Brusatol treatment as shown in Fig. 7B. Similar results were obtained using the in vitro $\mathrm{HK}-2 \mathrm{H} / \mathrm{R}$ cell model. The results demonstrated that $H / R$ treatment markedly reduced
HK-2 cell viability. SEV pretreatment increased cell viability in a dose-dependent manner, however, its protective effect was significantly reduced when the cells were also treated with brusatol (Fig. 7C). Western blot also confirmed that Nrf2 protein expression level in HK-2 cells was markedly reduced by brusatol treatment (Fig. 7D). These results indicated that SEV may exert renal protection, which is dependent on the activation of Nrf2.

\section{Discussion}

IR/hypoperfusion leads to the occurrence of oxidative stress and can lead to AKI. The pathophysiological mechanism of renal IR injury is complex and involves numerous pathways and factors, including the overproduction of oxygen free radicals, 
calcium overload, lipid peroxidation injury, the inflammatory response and cell apoptosis $(6,25)$. The overproduction of ROS serves an important role in AKI initiation and progression. Xanthine dehydrogenase is transformed into xanthine oxidase during ischemia and the resulting production of free radicals causes damage (26). Furthermore, following the reconstruction of the blood supply to ischemic organs, a large quantity of fresh oxygen flows in and produces excessive ROS, which oxidizes and modifies carbohydrates, proteins, lipoproteins and nucleic acids in tissues or cells, directly damaging their normal functions and leads to cell death $(27,28)$. This explains why caspase-3 is significantly upregulated in a mouse model of IR. Caspase-3 is the most common apoptotic signaling pathway and serves a key role in cell apoptosis (29). The present study demonstrated that SEV preconditioning decreased caspase- 3 protein expression levels in renal tubular epithelial cells induced by renal IR injury, which is one of the major mechanisms of the renal protective effect.

The results of the present study also demonstrated that following IR, oxidative stress in mouse kidneys was significantly upregulated and SEV preconditioning significantly reduced MDA and MPO levels, which may be related to SEV preconditioning resulting in increased activities of the antioxidant enzymes SOD and CAT. SOD is considered to be the most powerful antioxidant in the cell (30). CAT is a common antioxidant enzyme present in almost all living tissues that utilize oxygen (22). Both SOD and CAT are important endogenous antioxidant enzymes that act as components of the first line of defense against ROS. In the present study, the activation of SOD and CAT may have resulted from the upregulation of Nrf2 protein expression levels. Furthermore, immunohistochemistry demonstrated that SEV preconditioning significantly activated Nrf 2 protein expression level in the kidney tissues impaired by IR. Nrf2 is a basic leucine zipper stress-responsive transcription factor that maintains cellular redox homeostasis. Nrf 2 is a key regulator of the cellular redox response and acts as a signal for ROS scavenging (31).

Kelch-like ECH-associated protein 1 (Keap1) exists in the cytoplasm and binds with Nrf2, which mediates Nrf2 inhibition. When in vivo oxidative stress increases, Nrf2 dissociates from the Keapl protein and translocates to the nucleus, binds with ARE and induces the increased expression of Nrf2-regulated genes (e.g., HO-1, GST, NQO-1) (32).

Nrf2/ARE/HO-1 is one of the classic antioxidant signal axes. Ruan et al (33) reported that inhibiting Nrf2/HO-1 reduces the activities of SOD and GSH, and induces serious oxidative damage. Moreover, activation of the Nrf2/ARE/HO-1 signaling pathway can improve oxidative stress damage caused to cells. This conclusion is also confirmed in the present study. However, the effect of SEV on nitrification stress was not investigated in the present study. A follow-up study can further clarify whether SEV preconditioning involves the anti-nitrification stress mechanism. Leukocyte-endothelial cell adhesion is induced by proinflammatory factors during the course of inflammatory responses. When serum proinflammatory factors are dominant and the level of anti-inflammatory factors is relatively low, the inflammatory response will be amplified. Moreover, the incomplete repair of renal tubules and a continuous inflammatory reaction in tubulointerstitial tissues often occurs following renal IR, which can cause renal tissue fibrosis (34). The present study demonstrated that SEV preconditioning improved the post-IR fibrosis of renal tubules in mice. It can therefore be hypothesized that this slowing effect is mainly due to decreased inflammation and oxidative stress, whereby the activation of the $\mathrm{Nrf} 2 / \mathrm{HO}-1$ signaling pathway by $\mathrm{SEV}$ is one of the underlying mechanisms.

Oxidative stress triggers inflammation. TNF- $\alpha$ is one of the important inflammatory mediators in the inflammatory response. It can activate neutrophils and lymphocytes, increase the permeability of vascular endothelial cells, and regulate the metabolic activities of other tissues $(35,36)$. TNF- $\alpha$ can also promote the synthesis and release of other cytokines. TNF- $\alpha$ has a direct cytotoxic effect on renal intrinsic cells and induces apoptosis and cell death, whereby $\mathrm{TNF}-\alpha$ activates NADPH oxidase and induces ROS and cell damage (35). IL-6 can induce $\mathrm{T}$ cell activation, proliferation and differentiation, and participates in the immune response (37). IL-6 and other inflammatory factors are promoters of the inflammatory response $(35,37)$. In the present study, renal IR resulted in the significant upregulation of inflammatory factor expression, which may be mainly caused by the oxidative stress response in the renal tissue.

With the increase of clinical application, researchers found that the role of SEV in human body has two aspects: Potential cognitive damage and potential vascular protection $(38,39)$. However, further investigation is required to determine its function on different organs and pathways to fully understand the mechanism involved. The present study also demonstrated that SEV preconditioning significantly inhibited the production of chemokines, including MCP-1, ICAM-1 and VCAM-1 induced by IR in vivo. A previous study has demonstrated that chemokines serve important roles in IR-induced AKI (40). For example, knocking down ICAM-1 using short hairpin RNA significantly protects renal function (31) and inhibiting MCP-1 improves renal function in IR mice (16,31). MCP-1 is a key chemokine involved in regulating monocyte migration and infiltration. Previous studies $(41,42)$ have reported that increased MCP-1 expression recruits macrophages. The uptake of apoptotic cells by macrophages promotes tolerance by suppressing the release of proinflammatory cytokines and increasing the release of anti-inflammatory cytokines, such as TGF- $\beta 1(43,44)$, a key pro-fibrosis mediator.

The potential translational outcome of the present research is that SEV preconditioning may benefit patients with chronic kidney disease undergoing surgery and may become a standard treatment strategy before surgery for patients with chronic kidney disease in the future. However, the limitation of the present study is that it is an animal study, not a human one; therefore, it is not fully consistent with the human physical condition. Therefore, care must be taken to translate these findings into clinical practice.

In conclusion, the present study indicated that renal IR may lead to a significant increase in the oxidative stress response and the upregulation of the cellular inflammatory response. Following SEV preconditioning, the renal injury of IR mice was significantly improved due to the antiapoptotic, antioxidation and anti-inflammatory properties of SEV. Moreover, the results suggested that the upregulation of Nrf2 expression may contribute towards the renal protective effect of SEV in IR. 


\section{Acknowledgements}

Not applicable.

\section{Funding}

The study was supported by Beijing Natural Science Foundation (grant no. 7202138).

\section{Availability of data and materials}

The datasets used and/or analyzed during the present study are available from the corresponding author on reasonable request.

\section{Authors' contributions}

WXW and YBS were responsible for the conception and design of the study. WXW, ZRZ and YB were responsible for the acquisition and analysis of data. YXL and XNG conducted pathological analysis and evaluation. SZ performed TUNEL and in vitro hypoxia/reoxygenation analysis. YBS drafted the manuscript. SZ and YBS revised the manuscript. WXW and YBS confirm the authenticity of all the raw data. All authors read and approved the final manuscript.

\section{Ethics approval and consent to participate}

The animal experiment was approved by the Ethics Committee of Laboratory Animals of Chengde Medical College (Hebei, China; approval no. P2020145).

\section{Patient consent for participation}

Not applicable.

\section{Competing interests}

The authors declare that they have no competing interests.

\section{References}

1. Verma S and Kellum JA: Defining acute kidney injury. Crit Care Clin 37: 251-266, 2021.

2. Bidani A and Churchill PC: Acute renal failure. Dis Mon 35: 57-132, 1989

3. Kulvichit W, Kellum JA and Srisawat N: Biomarkers in acute kidney injury. Crit Care Clin 37: 385-398, 2021.

4. Yang L, Xing G, Wang L, Wu Y, Li S, Xu G, He Q, Chen J, Chen M, Liu X, et al: Acute kidney injury in China: A cross-sectional survey. Lancet 386: 1465-1471, 2015.

5. Hilton R: Acute renal failure. BMJ 333: 786-790, 2006.

6. Han SJ and Lee HT: Mechanisms and therapeutic targets of ischemic acute kidney injury. Kidney Res Clin Pract 38 427-440, 2019.

7. Jan R and Chaudhry GE: Understanding apoptosis and apoptotic pathways targeted cancer therapeutics. Adv Pharm Bull 9: 205-218, 2019

8. Chatterjee PK, Todorovic Z, Sivarajah A, Mota-Filipe H, Brown PA, Stewart KN, Cuzzocrea S and Thiemermann C: Differential effects of caspase inhibitors on the renal dysfunction and injury caused by ischemia-reperfusion of the rat kidney. Eur J Pharmacol 503: 173-183, 2004.

9. Xiao Z, Shan J, Li C, Luo L, Lu J, Li S, Long D and Li Y: Mechanisms of cyclosporine-induced renal cell apoptosis: A systematic review. Am J Nephrol 37: 30-40, 2013.
10. Wu TJ, Hsieh YJ, Lu CW, Lee CJ and Hsu BG: Linagliptin protects against endotoxin-induced acute kidney injury in rats by decreasing inflammatory cytokines and reactive oxygen species. Int J Mol Sci 22: 11190, 2021.

11. Vomund S, Schäfer A, Parnham MJ, Brüne B and von Knethen A: $\mathrm{Nrf2}$, the master regulator of anti-oxidative responses. Int J Mol Sci 18: 2772, 2017.

12. Abdo S, Zhang SL and Chan JS: Reactive oxygen species and nuclear factor erythroid 2-related factor 2 activation in diabetic nephropathy: A hidden target. J Diabetes Metab 6: 10.4172/2155-6156.1000547, 2015.

13. Ishii T, Itoh K, Takahashi S, Sato H, Yanagawa T, Katoh Y, Bannai S and Yamamoto M: Transcription factor Nrf2 coordinately regulates a group of oxidative stress-inducible genes in macrophages. J Biol Chem 275: 16023-16029, 2000.

14. Bolignano D, Donato V, Coppolino G, Campo S, Buemi A, Lacquaniti A and Buemi M: Neutrophil gelatinase-associated lipocalin (NGAL) as a marker of kidney damage. Am J Kidney Dis 52: 595-605, 2008.

15. Rhee H, Shin N, Shin MJ, Yang BY, Kim IY, Song SH, Lee DW, Lee SB, Kwak IS and Seong EY: High serum and urine neutrophil gelatinase-associated lipocalin levels are independent predictors of renal progression in patients with immunoglobulin A nephropathy. Korean J Intern Med 30: 354-361, 2015.

16. Albert C, Zapf A, Haase M, Röver C, Pickering JW, Albert A, Bellomo R, Breidthardt T, Camou F, Chen Z, et al: Neutrophil gelatinase-associated lipocalin measured on clinical laboratory platforms for the prediction of acute kidney injury and the associated need for dialysis therapy: A systematic review and meta-analysis. Am J Kidney Dis 76: 826-841.e1, 2020.

17. Naunova-Timovska S, Cekovska S, Sahpazova E and Tasić V: Neutrophil gelatinase-associated lipocalin as an early biomarker of acute kidney injury in newborns. Acta Clin Croat 59: 55-62, 2020.

18. Albeladi FI and Algethamy HM: Urinary neutrophil gelatinase-associated lipocalin as a predictor of acute kidney injury, severe kidney injury, and the need for renal replacement therapy in the intensive care unit. Nephron Extra 7: 62-77, 2017.

19. Jose RL, Damayanathi D, Unnikrishnan KP and Suneel PR: A comparison of sevoflurane versus sevoflurane-propofol combination on renal function in patients undergoing valvular heart surgery-A prospective randomized controlled pilot study. Ann Card Anaesth 24: 172-177, 2021.

20. Li H, Weng Y, Yuan S, Liu W, Yu H and Yu W: Effect of sevoflurane and propofol on acute kidney injury in pediatric living donor liver transplantation. Ann Transl Med 7: 340, 2019.

21. Ministry of Science and Technology of the PRC. Available from: https://www.puh3.net.cn/images/dwsyzx/zc/2020/5/30/227A82A 9E2B44976986A1EB84BB39836.pdf.

22. Zhang S, Xin H, Li Y,Zhang D, Shi J, Yang J and Chen X: Skimmin, a coumarin from hydrangea paniculata, slows down the progression of membranous glomerulonephritis by anti-inflammatory effects and inhibiting immune complex deposition. Evid Based Complement Alternat Med 2013: 819296, 2013.

23. Zhang S, Ma J, Sheng L, Zhang D, Chen X, Yang J and Wang D: Total coumarins from hydrangea paniculata show renal protective effects in lipopolysaccharide-induced acute kidney injury via anti-inflammatory and antioxidant activities. Front Pharmacol 8: 872, 2017.

24. Han SJ, Kim JH, Kim JI and Park KM: Inhibition of microtubule dynamics impedes repair of kidney ischemia/reperfusion injury and increases fibrosis. Sci Rep 6: 27775, 2016.

25. Gonul Y, Ozsoy M, Kocak A, Ozkececi ZT, Karavelioglu A, Bozkurt MF, Cartilli O, Keles I, Kocak H and Celik S: Antioxidant, antiapoptotic and inflammatory effects of interleukin-18 binding protein on kidney damage induced by hepatic ischemia reperfusion. Am J Med Sci 351: 607-615, 2016.

26. Linas SL, Whittenburg D and Repine JE: Role of xanthine oxidase in ischemia/reperfusion injury. Am J Physiol 258: F711-F716, 1990.

27. Granger DN and Kvietys PR: Reperfusion injury and reactive oxygen species: The evolution of a concept. Redox Biol 6: 524-551, 2015.

28. Granger DN, Rutili G and McCord JM: Superoxide radicals in feline intestinal ischemia. Gastroenterology 81: 22-29, 1981.

29. Nuñez G, Benedict MA, Hu Y and Inohara N: Caspases: The proteases of the apoptotic pathway. Oncogene 17: 3237-3245, 1998.

30. Ighodaro OM and Akinloye OA: First line defence antioxidants-superoxide dismutase (SOD), catalase (CAT) and glutathione peroxidase (GPX): Their fundamental role in the entire antioxidant defence grid. Alex J Med 54: 287-293, 2018. 
31. Silva-Islas CA and Maldonado PD: Canonical and non-canonical mechanisms of Nrf2 activation. Pharmacol Res 134: 92-99, 2018.

32. Loboda A, Damulewicz M, Pyza E, Jozkowicz A and Dulak J: Role of Nrf2/HO-1 system in development, oxidative stress response and diseases: An evolutionarily conserved mechanism. Cell Mol Life Sci 73: 3221-3247, 2016.

33. Ruan H, Luo J, Wang L, Wang J, Wang Z and Zhang J: Sika deer antler protein against acetaminophen-induced nephrotoxicity by activating Nrf2 and inhibition FoxO1 via PI3K/Akt signaling. Int J Biol Macromol 141: 961-987, 2019.

34. Meng XM, Nikolic-Paterson DJ and Lan HY: Inflammatory processes in renal fibrosis. Nat Rev Nephrol 10: 493-503, 2014.

35. Sun L and Kanwar YS: Relevance of TNF- $\alpha$ in the context of other inflammatory cytokines in the progression of diabetic nephropathy. Kidney Int 88: 662-665, 2015.

36. Griffin GK, Newton G, Tarrio ML, Bu DX, Maganto-Garcia E, Azcutia V, Alcaide P, Grabie N, Luscinskas FW, Croce KJ and Lichtman AH: IL-17 and TNF- $\alpha$ sustain neutrophil recruitment during inflammation through synergistic effects on endothelial activation. J Immunol 188: 6287-6299, 2012.

37. Tanaka T, Narazaki $M$ and Kishimoto T: IL-6 in inflammation, immunity, and disease. Cold Spring Harb Perspect Biol 6: a016295, 2014

38. Neag MA, Mitre AO, Catinean A and Mitre CI: An overview on the mechanisms of neuroprotection and neurotoxicity of isoflurane and sevoflurane in experimental studies. Brain Res Bull 165: 281-289, 2020.
39. Fang FQ, Sun JH, Wu QL, Feng LY, Fan YX, Ye JX, Gao W, He GL and Wang WJ: Protective effect of sevoflurane on vascular endothelial glycocalyx in patients undergoing heart valve surgery: A randomised controlled trial. Eur J Anaesthesiol 38: 477-486, 2021.

40. Sen Z, Jie M, Jingzhi Y, Dongjie W, Dongming Z and Xiaoguang C: Total coumarins from hydrangea paniculata protect against cisplatin-induced acute kidney damage in mice by suppressing renal inflammation and apoptosis. Evid Based Complement Alternat Med 2017: 5350161, 2017.

41. Chung CH, Fan J, Lee EY, Kang JS, Lee SJ, Pyagay PE, Khoury CC, Yeo TK, Khayat MF, Wang A and Chen S: Effects of tumor necrosis factor- $\alpha$ on podocyte expression of monocyte chemoattractant protein-1 and in diabetic nephropathy. Nephron Extra 5: 1-18, 2015.

42. Haller H, Bertram A, Nadrowitz F and Menne J: Monocyte chemoattractant protein-1 and the kidney. Curr Opin Nephrol Hypertens 25: 42-49, 2016

43. Voll RE, Herrmann M, Roth EA, Stach C, Kalden JR and Girkontaite I: Immunosuppressive effects of apoptotic cells. Nature 390: 350-351, 1997.

44. Fadok VA, Bratton DL, Konowal A, Freed PW, Westcott JY and Henson PM: Macrophages that have ingested apoptotic cells in vitro inhibit proinflammatory cytokine production through autocrine/paracrine mechanisms involving TGF-beta, PGE2, and PAF. J Clin Invest 101: 890-898, 1998.

This work is licensed under a Creative Commons Attribution-NonCommercial-NoDerivatives 4.0 International (CC BY-NC-ND 4.0) License. 